\title{
Marketing digital en universidades privadas en el estado Zulia
}

\author{
Digital marketing \\ in Zulia state private universities
}

\author{
Angelina S. Sosa Tota (Venezvela) \\ Magister en Gerencia de Empresas mensión Mercadeo \\ Universidad Rafael Belloso Chacín \\ sosa.angelina@gmail.com
}

\section{Resumen}

El siguiente trabajo de investigación tuvo como objetivo analizar el marketing digital en las universidades privadas en el municipio Maracaibo en el estado Zulia. El sustento teórico se fundamentó en los postulados de Kotler y Lane (2012), Kotler y Armstrong (2008), Ros (2008), Liberos (2013) y Muñiz (2010). Fue una investigación de tipo analítica, el diseño de investigación no transaccional-experimental, sustentada en la aplicación de una entrevista semiestructurada, guiada a los responsables en las universidades privadas en el municipio Maracaibo aplicar marketing digital. Los resultados obtenidos indicaron que el sector se ve afectado por la elevada inflación en Venezuela, ya que cada vez es más costoso adquirir equipos tecnológicos que faciliten a las universidades realizar actividades y estrategias de marketing digital.

\author{
Ma Cristina Useche Aguirre (Venezuela) \\ Doctorado en ciencias económicas \\ Universidad del Zulia \\ mariauseche@yahoo.es
}

\section{Summary}

The following research aims to analyze digital marketing in Zulia's state Maracaibo municipality private universities. Theoretical sustenance was based on Kotler and Lane (2012), Kotler and Armstrong (2008), Ros (2008), Liberos (2013) and Muñiz (2010) postulates. It was an analytical, non-transactional-experimental research design, based on a semi-structured interview, addressed to Maracaibo private universities digital marketing responsible. Obtained results indicated that sector is affected by Venezuela's high inflation, since it is becoming increasingly expensive to acquire technological equipment that facilitates universities to carry out digital marketing activities and strategies.
Recibido:

Evaluado:

Aceptado:
31 de julio de 2016

8 de abril de 2017

10 de junio de 2017

\section{PARA CITAR ESTE ARTíCULO/TO CITE THIS ARTICLE}

Sosa Tota, A. S. y Useche Aguirre, M. C. (2017). Marketing digital en universidades privadas en el estado Zulia, Poliantea 13(24), pág. ???-?? 
Se concluye que el direccionamiento se basa en buscar fidelidad de los clientes y para ello usan correctamente los medios de marketing digital, pero las herramientas empleadas solo se usan para informar las actividades internas de las instituciones, más no se ha fomentado la utilización de dichas herramientas, porque no aplican campañas vía correo electrónico para público externo, no utilizan blog interactivo y la fidelización online no es tomada en cuenta.

Palabras Clave: Marketing digital, medios, herramientas, usuarios, universidades privadas.
It is concluded that routing is based on seeking customer loyalty, correctly using digital marketing media, but tools used are only to inform the institutions internal activities, but said tools use of has not been encouraged, because they do not campaign via email, do not use interactive blog and online loyalty is not considered.

Keywords: Digital marketing, means, tools, users, private universities. 


\section{Introducción}

En el siglo XXI la innovación tecnológica en cuanto a computación, información y comunicación ha evolucionado a gran velocidad, transformando la civilización a una era digital, con características diferentes al siglo XX. Esta importante evolución se ve reflejada en Latinoamérica de manera significativa, según ComScore (2015) Latinoamérica represa el $10 \%$ de toda la audiencia de internet a nivel global, mostrando un crecimiento del $17 \%$ respecto a la cifras del año 2013, a su vez los usuarios dedican 28 minutos en cada visita superando el promedio de tiempo de visitas a nivel global y un $60 \%$ de los visitantes son menores de 35 años.

De acuerdo a Conatel (2015), Venezuela reporta que existen 15 millones de usuarios conectados representando un $58,94 \%$ de la población, siendo así uno de los países más destacados en Latinoamérica en lo que penetración en internet se refiere. Los datos estadísticos ratifican el constante crecimiento y acceso a internet, lo que se transforma en un elemento clave a considerar para las organizaciones e instituciones que estén interesadas en participar en el mercado. Los datos estadísticos ratifican el constante crecimiento y acceso a internet, transformándose en un elemento clave a considerar para las organizaciones e instituciones que estén interesadas en participar en el mercado.

Según una infografía publicada por tendencias digitales sobre internet en Latinoamérica (2015) Venezuela tiene el mayor índice de uso de internet en Latinoamérica reportando un $88,9 \%$, el uso más común es para correos con un $89 \%$, redes sociales un $85 \%$, noticias $83 \%$ y videos un $81 \%$ siendo la potencia viral con mayor porcentaje son las redes sociales con un $60 \%$. Lo antes plasmado representa la tendencia actual de buscar, compartir y socializar por medio de internet, razón por la cual las organizaciones e institución deben estar presente en dicho medio para poder crear una relación son sus clientes actuales y potenciales.

En este sentido, el sector de la educación universitaria se ha adaptado a estos cambios, adecuando sus enseñanzas a las 
necesidades de cada tiempo y a las demandas de la sociedad. Al respecto $\mathrm{Al}-$ barrán (2012), manifiesta que son múltiples los logros alcanzados en materia educativa tecnológica en Venezuela, pues se ha logrado incorporar el paradigma científico-tecnológico, no sólo a nivel de las labores educativas y de aprendizaje, sino también para agilizar la gestión de las instituciones educativas, a fin de incorporarlas efectivamente a la era digital, y en correspondencia a la incorporación de la convergencia digital en la vida de los estudiantes, quienes son denominados como Generación $\mathrm{Y}$, por Piscitelli (2009) ó Millennials por Oblinger (2003) ó nativos digitales por Cabra y Marciales (2009, p. 324) y son considerados como "hablantes nativos del lenguaje digital de los computadores, los videojuegos y la Internet" pues según Linne (2014, p. 217) se han "(...) criado en entornos digitales, son una generación post-mail, despliegan un mayor superposicionamiento de momentos off $\mathrm{y}$ online, $\mathrm{y}$ expresan de modo recursivo y cotidiano aspectos íntimos y experiencias estético-emotivas (...).

En el caso de las universidades privadas en el municipio Maracaibo, se pudo conocer a través de entrevistas realizadas, a los responsables de las actividades de mercadeo, Rodríguez (2014) de la Universidad Católica Cecilio Acosta, y Hernández (2013) de la Universidad José Gregorio Hernández, que se han plegado a la era digital, incorporando paulatinamente los mismos, tanto en los procedimientos administrativos internos, labores de consulta e información y mercadeo.
En tal sentido, estas instituciones han incorporado algunas modalidades de contacto con los actores educativos, es decir, equipo directivo, estudiantes, docentes y personal administrativo, utilizando medios digitales tales como Página Web, Facebook, Twitter, entre otros. Tales medios no solo han servido para establecer una comunicación interactiva, sino también para ejecutar acciones de publicidad de los servicios ofrecidos, así como labores de marketing digital.

En las actividades de mercadeo de las universidades privadas, el uso de algunos medios y herramientas ha contribuido en sus labores de mercadeo, permitiendo llegar a sus públicos objetivos, a través de comunicaciones directas, personales e interactivas. Indican que entre las causas que dan origen al problema, se encuentra la necesidad de incorporarse al mundo digital en forma efectiva, especialmente porque las universidades requieren mantener informados a sus usuarios de las actividades que llevan a cabo, y el uso de la publicidad a través de los medios de comunicación de masas resulta altamente onerosa en vista que su la gran mayoría de su público estudiantil es joven y están estrechamente relacionados al mundo digital y el uso de sus medios y herramientas para comunicarse, adquirir información y realizar procesos administrativos, entre otros.

Respecto al mercadeo digital se han realizado inversiones en equipamiento y tecnológica blanda en esta materia; sin embargo, reportan no haber alcanzado el desarrollo proyectado, por lo cual se requiere de estudios que permitan 
medir la opinión del personal responsable sobre herramientas del marketing digital, quienes se traducen en los gestores de las acciones digitales iniciadas por las universidades, a fin de comprender de forma más objetiva, el contexto situacional de las universidades privadas en el municipio Maracaibo del estado Zulia, para conocer las el entorno, así como la mezcla de marketing digital, los medios utilizados y las herramientas del marketing digital.

En tal sentido, se han definido medios para aplicar el marketing digital por parte de las universidades privadas, sin embargo, no se ha emprendido una valoración por parte del personal responsable del marketing digital, involucrados en esta actividad de planificación y ejecución de los mismos, lo cual genera deficiencias de información para llevarlo a la práctica y cumplir con los objetivos trazados por la institución.

Asimismo, se desconoce si se realiza un manejo apropiado por parte del personal responsable sobre herramientas del marketing digital, indicando falta información o competencias suficientes, por lo que, no están en condiciones de identificar y utilizar los vehículos más indicados, así como tampoco las modalidades más apropiada, a saber: uso del correo electrónico, publicidad digital, fidelización on-line o el empleo del blogging interactivo, entre otros, para el logro de sus objetivos.

La falta de dinamismo en el manejo de los contenidos de marketing falta puede ocasionar que las instituciones no avancen en el mercado, afectando su imagen institucional, esto limita las oportunidades de introducir los correctivos necesarios, así como las mejoras en forma oportuna, a los fines de optimizar sus labores en materia de marketing digital, lo cual coloca a las universidades privadas en una condición competitiva vulnerable, frente a instituciones que constituyen su competencia en el mercado, razón por la cual se requirió analizar el marketing digital en las universidades privadas en el municipio Maracaibo del estado Zulia, para ello se determinó el direccionamiento del marketing, se caracterizó la mezcla de marketing digital, se identificó los medios utilizados y las herramientas empleadas de marketing digital empleadas por las universidades privadas, aspectos que se presentan en los siguientes espacios de este trabajo.

\section{Metodología de la Investigación}

En cuanto al tipo de investigación, este estudio se clasifica como analíticodescriptivo, ya que se contrasta una serie de eventos con la teoría existente y se recolecta información que permite describir las herramientas del marketing digital empleadas, así como el direccionamiento y medios empleados; asimismo, se implementó un diseño de investigación no experimentaltranseccional, dado que se presentaron los datos sin modificación alguna en el período de la investigación comprendida entre enero 2014 - enero 2015, tiempo suficiente para desarrollar todas las fases del proceso de investigación.

La población del presente estudio estuvo conformada por las 4 universi- 
dades privadas ubicadas físicamente en el municipio Maracaibo registradas en el Ministerio del Popular para la Educación Universitaria (2013), a saber: Universidad Rafael Belloso Chacín (URBE), Universidad Rafael Urdaneta (URU), Universidad Católica Cecilio Acosta (UNICA) y Universidad Dr. José Gregorio Hernández (UJGH), los agentes informantes estuvieron representados por los departamentos de dirección de comunicaciones, dirección de relaciones institucionales y dirección de información y cultura, dichos informantes fueron los encargados de facilitar la información requerida para el abordaje de la variable de estudio.

La técnica de recolección de datos se basó en fuentes primarias, mediante entrevistas semi-estructurada, guiada de forma abierta sobre la variable en el sector. Asimismo, se emplearon fuentes secundarias, para efectos de esta investigación se utilizaron textos bibliográficos relacionados con la variable de estudio, revistas especializadas en el área de mercadeo y trabajos de grado a nivel de maestría sobre la temática del sector. Una vez obtenidos los datos, a través de entrevistas, se procedió al estudio de la información para organizar, revisar, procesar y analizar los datos, mediante la elaboración de un diagrama de arco (Hurtado, 2010), para establecer relaciones de correspondencia que permitieran sustentar y argumentar el razonamiento y detectar similitudes o diferencias entre los datos de los agentes informantes, para luego discutir, argumentar y generar conclusiones.

\section{Revisión de marketing digital}

Kotler y Armstrong (2007) se refieren al marketing digital como conjunto de estrategias y acciones que realizan las empresas para establecer una relación con un usuario y fomentar la compra de sus productos o servicios, a través de medios y herramientas digitales para establecer una relación positiva y duradera con los clientes, quienes pueden estar conectados online con las organizaciones, lo cual permite crear una ventaja competitiva frente a otras organizaciones, ofreciendo valor agregado a sus productos o servicios a través de las alternativas digitales.

El marketing digital es una forma de mercadeo que utiliza medios tecnológicos, para proyectar la imagen de una marca, mediante la publicidad de productos y servicios de una empresa, negocio y/o organización en diferentes canales de internet, bien sean redes sociales, páginas web, radio online, plataformas de vídeo y televisión, entre otras.

Complementado lo señalado, para Saavedra y col (2013) los mercados cuentan con una gran variedad de plataformas que facilitan la comunicación con los usuarios y la posibilidad de alentarlos a interactuar con ellos, mediante la integración de las comunicaciones publicitarias y las distintas estrategias de marketing.

Uno de los aspectos importantes de la influencia del desarrollo tecnológico y el cambio de comportamiento de los usuarios en el marketing del siglo XXI 
es la manera como a través del marketing digital, la publicidad es más especializada e individualizada, adaptada a sus requerimientos y necesidades, facilitando al anunciante vincularse con sus clientes, es decir, se conoce mejor las necesidades del anunciante, con la finalidad de realizar impactos digitales mucho más certeros y en correspondencia con las metas establecidas.

Sobre este aspecto, Anato (2006) afirma que las tecnologías de la comunicación han revolucionado tanto la economía global como a las empresas durante las últimas décadas. El desarrollo y aplicación de los sistemas de computación ha hecho posible su aplicación en una amplia gama de funciones y actividades. En los años 1980 las computadoras personales constituyeron un medio confiable y económico de acceso a las nuevas tecnologías. A finales del siglo XX surgió Internet como un mecanismo que hace posible la interconexión de computadoras y el acceso a la información en multimedia y otras fuentes de conocimiento. Así, un importante número de personas es capaz de acceder a niveles de información y conocimiento sin precedentes.

Explica que en el mundo moderno muchas de las funciones del marketing han sido reorientadas en función de la aparición de conceptos nuevos como calidad total, reingeniería de procesos y just in time (justo a tiempo). Las funciones individuales de los miembros de una organización también están sufriendo transformaciones, al dejar de realizar tareas o actividades aisladas para hacer mayor énfasis en el cumplimiento de los procesos y su impacto en la capacidad de la organización para satisfacer las necesidades de sus clientes. Internet como un medio de acceso libre a la información puede ser utilizada como una parte integrante del concepto moderno de marketing.

Una visión más técnica es presentada por Ros (2008), para quien el marketing digital es la aplicación de tecnologías por medios electrónicos para fomentar las actividades de mercadeo a los fines de lograr la rentabilidad, retención de clientes, entrega de comunicaciones integradas y servicios en línea, enfocados en dar cobertura a las necesidades de los públicos objetivos, por lo cual se considera uno de los más capaces para producir efectos favorables sobre el mercado.

Del mismo modo, Muñiz (2010) expresa que el marketing digital consiste en el conjunto de estrategias, técnicas y operaciones que, coordinadas a través de las redes digitales, utilizadas con el propósito de aumentar las ventas de un producto o servicio. Asimismo, combina cada uno de los principios del marketing convencional con las oportunidades y posibilidades que brinda la interactividad sobre los productos y servicios ofrecidos por las organizaciones. La revolución tecnológica ha producido un conjunto de cambios en el mercado que implican el conocimiento de nuevos medios para aprovechar todo su potencial y llegar a la consecución de los objetivos en las organizaciones.

Por lo que, el marketing digital es considerado postmodernista, dado que presenta una serie de cambios atípicos al marketing moderno, a saber: se encuen- 
tra la hiperrealidad, es decir, aspectos que se relacionan con la virtualización de los productos y del consumo. La hiperrealidad en el marketing hace referencia a una simulación de la realidad construida con la tecnología existente y técnicas de marketing, permitiendo que el cliente viva una experiencia que antes era imposible como es el caso de los juegos de realidad virtual, en el cual el usuario puede olvidar temporalmente la diferencia entre el juego y la realidad o las relaciones amorosas creadas y sostenidas únicamente mediante Internet.

En ese marco, se gesta una generación de personas (nativos digitales) con competencias digitales, aprendizaje experiencial y activo; interactividad y $\mathrm{CO}^{-}$ laboración; inmediatez y conectividad, con las cuales experimentan mayor confort, uso y empatía con entornos digitales y son capaces de armonizar simultáneamente con varias identidades, mostrando distintos comportamientos de consumo dependiendo del producto o de la situación en que esto ocurre, dando cabida a la aparición de nuevas tipologías de usuarios (Skiba y Barton, 2006).

El marketing digital combina los principios del marketing convencional con las oportunidades y posibilidades que brinda Internet y la interactividad de los servicios que ofrece. La revolución tecnológica ha producido un conjunto de cambios en el mercado que implican el conocimiento de nuevos medios para aprovechar todo su potencial y llegar a la consecución de los objetivos en las empresas. El objetivo es com- ercializar productos y servicios que satisfagan las necesidades de los clientes y que a través de campañas online se consiga atraer a clientes potenciales hacia páginas web corporativas, tiendas online, portales generalistas, etc.

Entre las áreas del marketing digital incluye: el posicionamiento en buscadores, medición y análisis de tráfico a un website, campañas de e-mail marketing \& newsletters, publicidad pago por click en buscadores, desarrollo y diseño de sitios web, comercio electrónico, desarrollo de aplicaciones (intranets, extranets), marketing viral y buzz marketing, publicidad on line, networking, blogs, redes sociales, entre otros.

Todas las empresas competitivas del siglo XXI se han preparado para lograr los objetivos de marketing, usando las tecnologías de información y comunicación, dado las ventajas que representan, como: amplia información disponible, igualdad de oportunidades con menos recursos, mayor eficiencia de las inversiones económicas, innovación en tácticas, interactividad sin precedentes, entre otros aspectos particulares.

En concordancia con lo señalado, Van Peborgh (2010) agrega que en el caso de la Web, ésta se ha convertido en un ejemplo de fragmentación, en la cual la comunicación está diseñada para adaptarse a los diferentes comportamientos de consumo por lo cual los mismos no se pueden generalizar. Como consecuencia de un mercado híper-saturado de productos estandarizados, el usuario deja de ser el punto final del proceso de 
producción y consumo, se convierte en una de las etapas que lo conforma.

Por tanto, se observa que los autores antes citados coinciden en sus apreciaciones sobre el marketing digital, siendo considerado un conjunto de acciones coordinadas para promover la compra de productos y servicios, a través de la interactividad.

\section{Resultados de la Investigación del Mar- keting Digital en el Sector Universitario}

Se consultó a los entrevistados el tipo de información que transmiten a sus públicos por medio de la implementación de marketing digital, la totalidad de los entrevistados coincidieron que utilizan los medios del marketing digital para transmitir información de carácter informativo, para toda la comunidad universitaria sobre todo lo que se realiza en el campus universitario como: eventos, proceso de inscripción, actividades extras curriculares, charlas, campañas institucionales, entre otras, con el fin dar a conocer todo lo que se realiza en la universidad.

Para todas las instituciones es de suma importancia demostrar las acciones que se realizan, con el fin de captar nuevos clientes, en el caso particular de las universidades, dichas instituciones deben demostrar todos los servicios que ofrecen y actividades que realicen para que se conozca la gestión de la misma y al momento de atraer nuevos clientes, estos tengan la información suficiente para comparar y decidir cuál es mejor opción en el sector; por otra parte, es relevante informar las actividades internas para mejorar la calidad de servicio y no exista desconocimiento de actividades internas, a su vez crear preferencia de marca, así como el sentido de pertenencia.

Lo expuesto anteriormente, se apoya en los postulados de Muñiz (2010) quien considera que el marketing informa para aumentar las ventas, ya que el éxito del producto o servicio en el mercado se vincula con la información que reciben los clientes, a fin de entender cuáles beneficios proporciona, cómo funciona y el modo de obtenerlo; por lo cual el mercadeo por medio del marketing digital también llega al cliente enfocado en informar.

Así mismo, Kotler y Armstrong (2007) señalan que el marketing digital puede dirigirse a los clientes actuales y potenciales sobre los servicios de la organización, sea empresa o institución, esta información se enfoca en aclarar información, transmitir nuevas informaciones de interés, entre otros.

En referencia a los beneficios del marketing digital en la comunicación interactiva con sus clientes, la totalidad de los entrevistados coincidieron que el beneficio del marketing digital en la comunicación interactiva es la inmediatez, mayor retroalimentación, mayor comunicación, instantaneidad y mayor interacción. Todos los entrevistados coincidieron en que gracias a la implementación de los medios del marketing digital el estudiante no sufre en el proceso, ya que siempre está informado sobre la situación actual de la institución y ayuda a resolver problemas en muchos 
casos sin necesidad que el estudiante se traslade a la universidad.

Esto ratifica los postulados de Muñiz (2010), quien expresa que la comunicación interactiva es un elemento que caracteriza a las interrelaciones en la red digital, integra los contenidos, aumenta la posibilidad de llegar a los mercados seleccionados como metas y facilita el intercambio entre las partes gracias al beneficio de interacción e inmediatez que ofrece la comunicación interactiva.

Con respecto a promover la imagen corporativa por medio del marketing digital, tres (3) de los entrevistados manifestaron que es para posicionar la universidad y tener reconocimiento académico para diferenciarse de la competencia y uno (1) de los entrevistado expresó que el propósito de la institución de mostrar su imagen es para dar a conocer todo lo que se está haciendo dentro de la universidad para que el público se entere.

Actualmente, demostrar las actividades ejecutadas por las instituciones es de suma importancia, y el sector universitario valora visibilizar los avances en docencia, investigación y extensión, pues mediante la promoción de imagen corporativa por plataformas digitales pueden tener reconocimiento académico, y a su vez les permite posicionar a cada universidad en el sector educativo.

En este sentido, se corrobora lo planteado por Kotler y Armstrong (2008), quienes señalan que el marketing digital puede ser direccionado con el propósito de promover la imagen de una empresa o institución destacando los beneficios para el usuario. Anudado a ello, sirve para mostrar al cliente cambios en la imagen de la empresa, tanto a nivel de su identidad gráfica como asociada a los significados de la misma.

Al indagar si el marketing digital ha contribuido en incrementar la lealtad con el cliente, la totalidad de los entrevistados respondieron positivamente, ya que se ha creado un sentido de pertenencia y confianza permitiendo que aumenten los números de seguidores en los medios de marketing digital, se destaca que gracias al marketing digital tienen contacto con ex alumnos en el exterior.

Lo antes expresado, soporta los postulados de Kotler y Armstrong (2008), quienes afirman que la lealtad del cliente se define como el grado en el cual el mismo consumidor/usuario compra un bien o utiliza un servicio con alta frecuencia. Como es el caso en las universidad se puede apreciar cuando los estudiantes continúan ampliando sus estudios dentro de la misma casa de estudio es decir estudiar diplomados, postgrados, cursos entre otros. Los entrevistados en su totalidad comentaron que sus clientes continúan usando sus servicios con el pasar de los años luego de finalizar la carrera académica. Es de suma importancia resaltar la importancia que se debe otorgar a la lealtad del cliente, ya que permite que la empresa o institución crezca y permanezca en el mercado, ya que un cliente leal genera un flujo de ingresos constantes.

Asimismo, todos los entrevistados respondieron de forma afirmativa sobre la 
importancia de transmitir información acertada a los usuarios es reales y potenciales, basados en una comunicación interactiva y en la promoción de imagen de las instituciones para lograr la lealtad de los clientes, siendo así el direccionamiento centrales de aplicar marketing digital en las universidades privadas.

Cada vez son más las personas que pasan más tiempo conectados a internet, buscando información sobre los productos o servicios que desean adquirir, creando comunidades y estableciendo conversaciones con las marcas, motivo por el cual es clave la implementación de marketing digital en las empresas o instituciones, no estar presente en la red en la actualidad es sinónimo de no existir. Mostrar la actividad económica de las empresas con información acertada y una promoción de imagen digital adecuada logra captar la atención de clientes potenciales.

El direccionamiento del marketing digital aplicado en el sector universitario privado se basa en buscar fidelidad de los clientes, por medio de la comunicación interactiva, la constante actualización de informaciones de interés y en fortalecer la imagen de la institución por medio de la promoción de imagen, con el objetivo de posicionarse en el mercado para que futuros estudiantes sientan afinidad con la universidad.

En relación a la mezcla de marketing digital utilizada por las universidades privadas está compuesta por producto, precio, plaza y promoción y al aplicarlos al mundo digital se busca satisfacer a los usuarios es y que el servicio que se ofrece sea más sencillo. Uno de las características principales del marketing digital es la participación de los usuarios es en el proceso. Por otra parte, en cuanto a servicio ofrecido en el mundo digital por las universidades privadas se busca facilitar el proceso de estudio de los alumnos en base a otorgarles información de valor que ellos puedan utilizar para realizar las diferentes solicitudes que se presenten al momento que cada estudiante curse su carrera académica.

En referencia a los servicios ofrecidos a través del marketing digital tres (3) de los entrevistados manifestaron, horarios digitales, correo institucional, aplicación de consultas, inscripción en línea, biblioteca digital, revistas digitales y uno (1) de los entrevistados manifestó que solo ofrecen horarios, correo institucional, portal de noticias.

Lo antes expuesto ratifica lo expuesto por Kotler y Lane (2012) en que el producto o servicio representa la oferta tangible o intangible de la firma al mercado, incluyendo calidad, características, marca; entre otras, lo cual es presentado para satisfacer los requerimientos de los clientes. Para el caso de las universidades se oferta por medio de la implementación del marketing digital, cierta cantidad de servicios intangible que son de suma importancia para satisfacer sus necesidad que terminar su carrera académica.

En lo que respecta a los precios, es conveniente el uso del marketing digital para promover los servicios de la universidad, la totalidad de los entrevista- 
dos respondieron de manera afirmativa y expresaron que es más económico que utilizar los medios tradicionales (radio, presa, televisión, cine y revista), y a su vez el marketing tiene un valor agregado que es poder interactuar más con el público. A su vez, los entrevistados manifestaron los servicios ofrecidos por marketing digital no tienen valor económico extra para los estudiantes, a menos que sean materias a distancia que son casos especiales.

En base a los resultados, se confirma lo que expresa Muñiz (2010) sobre el marketing digital en referente a precio, en los medios digitales se tiene la posibilidad de comparar y validar más los atributos, lo que permiten tomar una decisión más informada. Al estar presentes en el mundo digital los usuarios potenciales pueden evaluar la relación entre costo y beneficio de una universidad con otra para así tomar una decisión.

En cuanto a la calidad de tecnología utilizada en el marketing digital, la totalidad de los entrevistaron manifestaron que siempre están en búsqueda de la mejor tecnología y equipos para poder implementarlo, y por los problemas económicos del país, no ha podido tener un mayor crecimiento en la adquisición de equipos actualizados como tablets, teléfonos inteligentes, routers entre otros, los cuales les facilitaría el trabajo. A su vez los entrevistados manifestaron que la disposición de sus servicios por medio del marketing digital se hace posible gracias a la tecnología utilizada y a los medios del marketing digital.
Según Kotler y Lane (2012), la plaza es la selección de canales comerciales a través de los cuales el producto o servicio llegará mercado adecuado. Para el caso particular de las universidades, dichas instituciones hacen llegar sus servicios por medio de una plataforma digital (Página web o redes sociales) y su plaza se convierte en dicha plataforma digital y la disposición de la información dentro de la misma.

Los resultados y la teoría en este punto se complementan, en vista que Muñoz (2010) expresa que la plaza aplicada al marketing digital promueve un mayor acercamiento entre el prestador del servicio y el usuario final, con menor número de intermediarios y también intervienen nuevos modelos de negocio, para entregarle al cliente una mayor satisfacción o experiencia de compra o uso.

Dicha satisfacción o experiencia mencionada por Muñiz (2010) se ve reflejada con el uso del usuario de un medio digital para conseguir información necesaria, esa experiencia se vive gracias a la calidad de tecnología que se utiliza, pese a los problemas económicos del país buscan utilizar tecnologías para ser eficaces en la implementación de marketing digital.

Al momento considerar los beneficios en materia promocional obtenidos, a través del marketing digital, tres (3) de los entrevistados manifestaron que entre los beneficios se encuentran: menor inversión en medios tradicionales, mayor visibilidad, mayor alcance y aumento de clientes. A diferencia de uno (1) de los entrevistados manifestó que el benefi- 
cio que pueden observar es que cada vez son más los estudiantes que solicitan información y la respuesta es más efectiva gracias a la implementación marketing digital.

Esto ratifica lo expresado por Kotler y Armstrong (2007) en cuanto a que la promoción comprende las diferentes actividades que la empresa realiza para comunicar las cualidades de sus productos o servicios. Estos autores señalan a su vez que la publicidad en las web sites constituye una herramienta de información sobre los atributos de productos y sus beneficios, que también ofrecen los medios tradicionales pero la ventaja es que la inversión es menor en un web site y este dura en el tiempo a diferencia de un medio tradicional.

La mezcla de marketing utilizada por las universidades privadas se adapta al entorno digital y se considera producto o servicio, precio, plaza y promoción desde la perspectiva del uso de la tecnología para poder llegar a los usuarios actuales y potenciales y lograr los objetivos del mercadeo.

El marketing evoluciona y se complementa de la mano de avances tecnológi$\cos$, la tradicional mezcla de marketing conformada por las $4 \mathrm{P}$ se adaptan al entorno digital para aprovechar los beneficios que ofrece el mundo digital, este amplia los medios y las herramientas para mercadear los productos o servicios de las organizaciones. En el marketing digital se debe pensar en los usuarioes y para ello se deben adaptar el mix tradicional al entorno digital para aprovechar las oportunidades que este brinda.

Sobre los medios utilizados en el marketing digital por las universidades privadas en el municipio Maracaibo del estado Zulia. Al momento de indagar sobre el propósito del uso de la página web, como medio de marketing digital, la totalidad de los entrevistados manifestaron que el propósito es tener la información más ampliada para toda la comunidad estudiantil, permitiendo tener dentro de mismo medio diferente tipos de informaciones, así como varios servicios al mismo tiempo (horario, pensum, inscripciones) de esta manera los diferentes tipos de públicos como: personal administrativo, estudiantes, personal obrero y público potencial se benefician de la página web.

El uso de la página web como medio del marketing digital ayuda a proveer información ampliada de los productos o servicios de una empresa o institución, los resultados se apoyan con los planteamientos de Liberos (2013) sobre la página web la cual permite, a través de hipervínculos obtener distintas informaciones sobre la organización. Para el caso de las universidades privadas, estas se benefician mucho de este medio, ya que aprovechan las bondades para mostrar su gestión de manera ampliada, publican actividades y eventos, muestran sus variedad de servicios, permite informar sobre el proceso de inscripción y notas, entre otros usos que le dan a esta herramienta.

Al indagar los propósitos de utilizar redes sociales como Facebook como 
medio de marketing digital, se observó que tres (3) de los entrevistados manifestaron que el uso del Facebook se debe a que gran parte de sus públicos utiliza Facebook y que su propósito principal del uso es la información permanece en el tiempo y queda como memoria gráfica, ya que permite publicar fotos, videos y mensajes siendo de gran utilidad para demostrar los eventos internos de la universidad. Por otra parte uno (1) de los entrevistados manifestó que no utilizan Facebook como medio para implementar marketing digital.

Lo antes mencionado corrobora los planteamientos de Matias (2011, p.14), quien afirma que Facebook es un medio "(...) de push y pull de información", a través del cual es posible contactar clientes actuales y ganar clientes potenciales y que para el caso particular de este medio cuenta con más de cuatrocientos millones de usuarios activos. Dicha herramienta es gratuita y permite compartir diferentes tipos de contenido, como video, fotos, textos, música entre otros.

Para manejar Facebook como empresa o institución se puede utilizar el Fanpage, que es un servicio que ofrece la red social. En vista que una de las redes con más seguidores se debe sacar provecho de esta red para potenciar las iniciativas de marketing digital.

Sobre el propósito del uso del twitter como medio de marketing digital, se pudo conocer que tres (3) de los entrevistados manifestaron que el objetivo principal del uso de twitter es: para informar, tener una comunicación más directa y poder interactuar más gracias a la rapidez que ofrece dicho medio. Por su parte uno (1) de los entrevistados manifestó que no utilizan twitter como medio para implementar marketing digital.

Al contrastar los resultados con la postura expuesta por Ramos (2013), se encuentra correspondencia, pues como red de información de tiempo real que permite compartir y descubrir lo que está pasando en el momento, a su vez es un servicio gratuito de microblogging, que hace las veces de red social permitiendo compartir mensajes cortos. El sector de las universidades privadas en su mayoría conoce los beneficios del uso del Twitter ya que manifiestan uno de sus beneficios que la comunicación directa y rápida.

En lo que respecta al propósito del uso de Instagram como medio de marketing digital, dos (2) de los entrevistados manifestaron que utilizan Instagram para informar y demostrar con imágenes lo que se realiza dentro de la institución y por ser la red más usada Portillo $(2014$, p.1) expresó:

\footnotetext{
"Instagram es la red más dinámica actualmente y permite publicar la realidad por medio de imágenes en el momento que ocurre la actividad, también se puede manejar todo lo referente a la felicitaciones y publicar imágenes con frases célebres, Instagram unifica el esfuerzo de las redes que utilizan".
}

Por otra parte, dos (2) de los entrevistados manifestaron que no utilizan Instagram al momento de implementar marketing digital. Lo arrojado por este ítem 
concuerda con lo indicado por Liberos (2013), quien señala que Instagram es una red social que permite transmitir fotografías de un producto o servicio en específico. Para utilizarlo como estrategia de marketing digital, además de medio informativo, se pueden realizar concursos, invitar a los seguidores a crear y compartir fotos, dar a conocer nuevos productos o servicios, usar etiquetas para el posicionamiento, pues el contenido visual atrae las emociones de una manera que el texto no puede.

Al indagar el propósito del uso de youtube, se observó que solo uno (1) de los entrevistados manifestó que no utilizan youtube en su gestión de marketing digital, mientras que los otros tres (3) de los entrevistados manifestaron que utilizan el canal de youtube para mostrar videos institucionales, de eventos, capsulas informativas sobre procesos internos de la universidad y su propósito es mostrar más ampliada la información en apoyo a las otras redes, ya que permite utilizar audio y video, esto corresponde con Liberos (2013), para quien los videos digitales emergen en este medio como un canal eficiente para campañas publicitarias, facilitando información y experiencias agradables.

Lo expuesto anteriormente permite inferir que las universidades privadas aplican correctamente los medios de marketing digital para llegar a sus clientes actuales y potenciales. Asimismo, usan los diferentes medios para informar todas las actividades internas de las instituciones para brindar un buen servicio y tener presencia en el mundo digital; dichos medios apoyan todos los esfuerzos que se realizan fuera del mundo online y apoyan el logro de los objetivos de comunicación e interacción con el cliente.

Estar presente donde se encuentran los usuarios en el mundo digital es clave para poder generar una conversación con ellos y conocer sus opiniones sobre los productos y servicios que se ofrecen. Las redes sociales actualmente ayudan a muchas empresas a compartir información de lo que ofrecen estableciendo vínculos, permitiendo el dialogo y el intercambio de ideas, donde los usuarios en este caso usuarios son los más importantes, ya que ellos determinan que le interesa de cada marca y demuestran sus descontento o no con cualquier actividad que realicen las marcas.

Con respecto, a las herramientas del marketing digital, utilizadas por las universidades privadas en el municipio Maracaibo del estado Zulia, se pudo indagar sobre la finalidad del correo electrónico como herramienta de marketing digital, dos (2) de los entrevistados manifestaron que utilizan el correo electrónico con la finalidad de responder solicitudes y consultas. Por otra parte, dos (2) de los entrevistados manifestaron que utilizan el correo electrónico solo para público interno para ampliar la información entre departamentos.

Las universidades privadas no explotan los beneficios que ofrece el correo electrónico como herramienta para realizar publicidad o informar a sus diferentes tipos de públicos, teniendo así un déficit en su gestión del marketing digital, esto en base a lo expuesto por los pos- 
tulados de Muñiz (2010) quien expresa que el correo electrónico utilizado como e- mail marketing es la utilización del correo electrónico con ánimo comercial o informativo y se diferencia de otras herramientas de marketing tradicional porque permite realizar una oferta personalizada y exclusiva a cada persona a través de un medio inmediato como es el correo electrónico. Es decir, los resultados no coinciden con la teoría, por lo que no se valida la pertinencia de ésta en la actividad comunicacional universitaria en el estado Zulia.

En referencia a los beneficios de la publicidad digital para la promoción de los servicios de la universidad tres (3) de los entrevistados manifestaron que entre los beneficios están, tener presencia en otros medios digitales por medio de Banner y mayor alcance que ofrece la publicidad digital. Uno (1) de los entrevistados manifestó que no poseen publicidad digital solo publicidad en medios impresos. Pese a que tres (3) de los entrevistados mostraron una repuesta positiva en referencia a la publicidad digital, no existe un plan estructurado donde apliquen de manera correcta dicha herramienta.

Los resultados coinciden con los postulados de Baggot (2012) quien expresa que cuando se extrapola la publicidad al entorno digital se está aplicando las herramientas publicitarias en los nuevos medios de comunicación aprovechando los nuevos formatos como publicidad en buscadores, banners, rich media ads, microsite, entre otros, potenciados además por la gran capacidad de segmentación que ofrece internet, gracias a la web 2.0 .

En referencia a la fidelización online se realizó la siguiente pregunta ¿Cómo miden la fidelización de los usuarios? La totalidad de los entrevistados manifestaron que no poseen una herramienta específica para validar la fidelización online, pero mediante el aumento diario de seguidores, los comentarios satisfactorios y el aumento de la participación en las redes permiten conocer la satisfacción de los clientes. Estando en era de la tecnología de la información y comunicación parece inaudito que la población en estudio no mida cuantitativamente la fidelización de los clientes, cuando eso es considerado una tarea comunicacional básica de toda organización; al respecto Chistopher y Wirtz (2004) la consideran como un objetivo esencial del mercadeo relacional que responde a la necesidad de vincular eficazmente el marketing, la calidad y el servicio para conseguir la satisfacción total del cliente y establecer relaciones a largo plazo. La fidelización de clientes representa uno de los aspectos indispensables que guían los esfuerzos del mercadeo. Por lo que, para el sector universitario privado es de suma importación utilizar la herramienta de fidelización online, ya que esto permite mantener, establecer y crear relaciones interinstitucionalmente en docencia, investigación y extensión.

Si bien no tienen una herramienta específica para medir la fidelización online, se encuentran iniciados en el camino, los resultados de todo el instrumento de recolección de datos permiten observar que la utilización de las redes sociales, como medio del marketing dig- 
ital les permite a las universidades crear comunidades virtuales, y es por eso que existe la participación de los usuarios, quienes demuestran su satisfacción o no sobre un tema de interés en la comunidad universitaria.

Lo anterior ratifica los postulados expuestos por Liberos (2013), quien expresa que para conseguir una fidelización a la web por parte del público se deben agrupar en función a su respuesta, luego se segmentan los clientes para formargrupos internamente lo más homogéneos posible. La creación de comunidades virtuales permite que se establezca la participación de los usuarios mediante el dialogo.

Sobre la utilización del blog interactivo y sus beneficios. Por unanimidad, el total de los entrevistados manifestaron que no utilizan la herramienta de blog interactivo como parte de la implementación de marketing digital. Es importante resaltar que al no utilizar esta valiosa herramienta pierden varios beneficios que los blog ofrecen como: mayor exposición en el mundo online y en los motores de búsqueda, es económico y fácil de usar, facilita relaciones con los clientes ya que las personas pueden dejar comentarios sobre los artículos o informaciones publicadas, permite ampliar la información que no está en la página web y no se puede transmitir por redes sociales, entre otros.

Esto no corresponde con lo expuesto con Harris y Rae (2009), para quienes los blogs permiten publicar interacciones con los usuarios. Las empresas están utilizando blog interactivo para la con- strucción de reputación de marca y relaciones con los clientes. Las empresas e instituciones que utilizan esta herramienta la visualizan como una oportunidad en el ejercicio del marketing, en pro de la consecución de sus objetivos. De lo anteriormente expuesto, se puede inferir que el uso de las herramientas del marketing digital aún no ha sido potenciado en su totalidad por parte del sector de educación universitaria privada.

El uso de las herramientas de marketing digitales es de vital importancia, ya que brindan excelentes oportunidades para realizar publicidad, incentivan la interacción con los clientes y promueve la adquisición de un producto o servicio. Las herramientas de marketing digital permiten transmitir rápidamente un mensaje comercial con un bajo presupuesto en vista que la utilización de medios y herramientas digitales son menos costosas que los medios tradicionales. Al no utilizar las bondades que ofrecen dichas herramientas se pierde una importante forma de hacer publicidad a menor costo y de llegar a más clientes potenciales.

\section{Conclusiones}

Con respecto al direccionamiento de marketing digital, el sector universitario privado se basa en buscar fidelidad de los clientes, por medio de la comunicación interactiva, la constante actualización de informaciones de interés y en fortalecer la imagen de la institución por medio de la promoción de imagen, con el objetivo de posicionarse en el mercado para que futuros estudiantes sientan afinidad con la universi- 
dad. Por otra parte, gracias a la inmediatez de la comunicación interactiva es posible responder las solicitudes de los participantes con mayor rapidez, existe mayor retroalimentación que en años atrás, cuando no existían los medios y herramientas digitales.

Uno de los propósitos más importantes del uso de medios y herramientas del marketing digital es la promoción de imagen de las instituciones y la lealtad del cliente. El sector universitario entiende que mediante la promoción de imagen corporativa mediante plataformas digitales pueden tener reconocimiento académico destacando las cualidades y los beneficios que ofrecen, a su vez les permite posicionar las universidades creándose un sentido de pertenencia y confianza permitiendo que aumenten los números de seguidores en los medios de marketing digital, por lo que, gracias al marketing digital tienen contacto con ex alumnos en el exterior.

En referente a la mezcla de marketing digital se pudo observar que la mezcla de mercadeo tradicional es utilizada por las universidades privadas y se adapta al entorno digital para poder llegar a los usuarioes actuales y potenciales y lograr los objetivos del mercadeo, como cada día surgen nuevos avances la mezcla de mercadeo debe estar en constante revisión para provechar los beneficios que ofrece el mundo digital y decidir si ampliar o no la mezcla de marketing.

En relación a los medios de marketing digital se concluye que las universidades aplican correctamente los medios de marketing digital para llegar a sus clientes actuales y potenciales. Utilizan los diferentes medios para informar todas las actividades internas de las instituciones para brindar un buen servicio y tener presencia en el mundo digital. Dichos medios apoyan todos los esfuerzos que se realizan fuera del mundo online y apoyan el logro de los objetivos de comunicación e interacción con el cliente.

Con relación a las herramientas de marketing digital, el sector universitario privado no ha fomentado la utilización de dichas herramientas, ya que no aplican campañas vía correo electrónico para público externo, no utilizan blog interactivo, la fidelización online no es tomada en cuenta, lo único que aplican a medias es la publicidad digital por medio de la utilización de banners electrónicos en páginas web, lo que ocasiona una pérdida de los beneficios que ofrecen. Al momento de aplicar marketing digital todo va en enlazado, los esfuerzos realizados en el marketing tradicional, las actividades internas, la utilización de medios y herramientas acordes al sector se deben trabajar de manera coordina y enfocada a lograr objetivos sin perder tiempo y recursos.

En base a la información recolectada en la presente investigación se evidencia que el sector se encuentra en un continuo aprendizaje sobre el marketing digital, aunque tienen dificultades económicas para adquirir equipos tecnológicos actualizados que les permita implementar herramientas de marketing digital.

El sector universitario privado por la naturaleza de su público debe man- 
tenerse en una constante actualización, para brindar los mejores servicios a los jóvenes que cada día se integran, desarrollan y amplían más las bondades del mundo digital, compartiendo sus experiencias, publicando mensajes contundentes a la sociedad y destacando las bondades y potencialidades de cada institución universitaria para consolidar las marcas en este sector privado tan competitivo nacional e internacionalmente.

\section{Referencias}

Albarrán, F. (2012). Logros de la Revolución Educativa y Tecnológica. Obtenida el 27/09/2013, de http://fundabit.me.gob.ve/noticias-del-ano-2012/octubre/392logros-de-la-revolucion-educativa-y-tecnologica.

Anato, Mercedes (2006), El uso de los instrumentos clásicos del marketing y la tecnología digital en turismo Estudios y Perspectivas en Turismo, Vol 15, núm 1, Argentina, Centro de Investigaciones y Estudios Turísticos. Disponible, en: http://www.redalyc.org/articulo. oa? id=180713884002. Consultado el 01/11/2013 Pp. 19-40.

Chávez, N. (2001). Introducción a la investigación educativa. Tercera edición. Maracaibo, Venezuela. Pp. 20-56.

ComScore (2015), Futuro Digital Latinoamérica 2015. Obtenido el 24/05/2015 de:

http://www.comscore.com/lat/ Prensa-y-Eventos/Blog/Futuro-
Digital-America-Latina-2015.

Chistopher, L. y Wirtz, J. (2004). Marketing Services. México: Editorial Prentice Hall. Pp. 20-41.

Comisión Nacional de Telecomunicaciones (CONATEL) (2015). 2015 comenzó con más de 3 mil millones de usuarios de Internet. Extraído de: www.conatel.gob.ve/ 2015-comenzo-con-mas-de-3mil-millones-de-usuarios-deinternet/.

Harris, L. y Rae, A. (2009). Social networks: the future of marketing for small business. Journal of Business Strategy. Vol. 30 Iss: 5, Pp.24 - 31.

Hernández, J. (2014). Entrevista. Universidad José Gregorio Hernández Fecha de la entrevista: 09/09/2014. Maracaibo. Estado Zulia. Venezuela.

Hurtado, Jacqueline (2010). Metodología de la Investigación Holística. Guía para la comprensión holística de la ciencia, 4ta. Edición. Bogotá, Colombia. Ediciones Quirón.

Hurtado, Jacqueline. (2010). El Proyecto de Investigación. Comprensión Holística de la Metodología y la Investigación. 6ta. Edición. Caracas, Venezuela. Bogotá, Colombia. Ediciones Quirón. Pp. 39-56, 97-165.

Kotler, P. y Lane K. (2012). Dirección de marketing. 14va Edición. México. Editorial Pearson Educación. 
Kotler, P. y Armstrong, G. (2008) Fundamentos de marketing. Traducción: Mónica Gabriela Martínez Gay. Octava Edición. Naucalpan de Juárez, México. Editorial Pearson Educación.

Kotler, P. (2007). Dirección de la Mercadotecnia. México. Editorial Prentice Hall. Pp. 01-775.

Kotler, P. \& Armstrong, G. (2007). Marketing para Latinoamérica. México: Editorial Pearson Educación. Pp. 01- 655.

Liberos, E. (2013). El libro del Marketing Interactivo y la Publicidad Digital. Madrid: ESIC Editorial. Pp. 01-492.

Linne, J. (2014). Dos generaciones de nativos digitales. Intercom - Revista Brasileira de Ciências da Comunicação, Julio-Diciembre, 203-221.

Marciales-Vivas, G. P., Cabra-Torres, F. (2009). Mitos, realidades y preguntas de investigación sobre los 'nativos digitales': una revisión. Universitas Psychologica, Mayo-Agosto, 323-338.

Matias, M. (2011). El Marketing Digital en la Empresa. Obtenida el 26/05/2015, de http://pqs.pe/sites/default/files/archivos/2015/aprende-mas/04/sbello/redes_sociales-el-marketing-digital-enla-empresa.pdf.

Ministerio del Popular Para la Educación Universitaria. (2013). Libro de Oportunidades de Estudio en las Instituciones de Educación Universitaria. Recuperado de: http://loeu.opsu.gob.ve/vistas/ instituciones/busquedaEstados.php Consultado el 15-102013.

Muñiz, R. (2010). Marketing XXI. Madrid: Editado por la Universidad a Distancia de Madrid. Pp. $1-422$.

Oblinger, D. (2003). Boomers, GenXers, Millennials. Understanding the new students. Educase, JulyAugust, 37-47.

Piscitelli, A. (2009). Nativos digitales. Buenos Aires: Santillana.

Portillo, R. (2014). Entrevista. Universidad Católica Cecilio Acosta. Fecha de Entrevista: 14/01/2014. Maracaibo. Estado Zulia. Venezuela.

Ramos, J. (2013). Twitter para Empresas. Obtenida el 26/05/2015, de http://www.freelibros.org/marketing/twitter-para-empresasjuanjo-ramos.html.

Ros, V. (2008). E- Branding. Posiciona tu Marca en la red. Madrid: Editorial Netbiblio, p. 1-250.

Rodríguez, A. (2014). Entrevista. Universidad Católica Cecilio Acosta. Fecha de entrevista: 11/09/2014. Maracaibo. Estado Zulia. Venezuela.

Saavedra, F; Rialp, J; Llonch, J. (2013). El uso de las redes sociales digitales como herramienta de marketing en el desempeño empre- 
sarial. Cuadernos de Administración, Bogotá: Pontificia Universidad Javeriana, Vol. 26, No. 47, Julio-diciembre. Pág. 205231, Obtenida el 27/05/2015, de http://www.redalyc.org/articuloBasic.oa?id=20531182009.

Skiba, D. y Barton, A. (2006). Adapting your teaching to accommodate the Net Generation of learners. OJIN: The Online Journal of Issues in Nursing. 1(2). 1-11.
Tendencias digitales. (2015). Infografía Usos de internet en Latinoamérica. Recuperado de: http://tendenciasdigitales.com/ 2000/infografia-usos-de-internet-en-latinoamerica/. Consultado el: 25/05/2015.

Van P. (2010). Odisea 2.0. Las marcas en los medios sociales. Argentina: La Crujia Ediciones, $\mathrm{p}$. 20-106. 
ANGELINA STEFANIA SOSA TOTA \& MARÍA CRISTINA USECHE AGUIRRE

26 | Poliantea | p. 26-26 | VOLUMen XiII | NÚMERO 24 | AGOSTO 2017 